\title{
SELF-TUNING FUZZY CONTROL OF ELECTRO-HYDRAULIC
}

\author{
SERVO SYSTEM
}

\author{
Takayoshi MUTO*, Ping YU**, Yoshiharu TAKEDA** and Hironao YAMADA* \\ ${ }^{*}$ Department of Mechanical Engineering, Faculty of Engineering \\ Gifu University \\ 1-1 Yanagido, Gifu, 501-11 Japan \\ ${ }^{* *}$ Graduate Student, Faculty of Engineering \\ Gifu University \\ 1-1 Yanagido, Gifu, 501-11 Japan
}

\begin{abstract}
This study deals with a self-tuning fuzzy controller (STFC) when it is applied to an electro-hydraulic servo system. The STFC has a hierarchic structure consisting of an algorithm being identical to a fuzzy controller at the lower loop, and a tuning algorithm accommodating the performance evaluation and parameter modification function at the upper loop. As an algorithm for tuning at the upper loop, another fuzzy reasoning is adopted for constructing a fuzzy selftuner. The whole procedure of the STFC is repeatedly performed until an acceptable level of control is achieved. The basic functions of the STFC can be summarized as follows: (a) to issue appropriate control action while evaluating the performance. (b) to modify the control action based on the evaluation. It is verified by experiment and digital simulation that the STFC developed in this study is useful and effective for the control of electro-hydraulic servo system.
\end{abstract}

\section{KEY WORDS}

Fluid Power System, Fuzzy Control, Hydraulic Servo System, Simulation, Self-Tuning Control

\section{NOMENCLATURE}

$A, B, C$ : Gains of error, change in error and control input

$a, b, c:$ Incremental factors for $A, B, C$

$D, D o$ : Damping ratio and its value of fuzzy support set

E, Eo : Overshoot ratio and its value of fuzzy support set

$e, e_{0}$ : Error of rotating velocity and its value of fuzzy support set

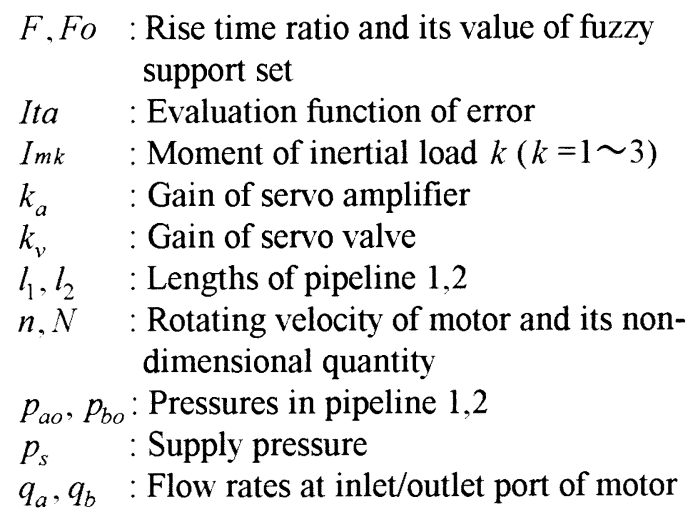




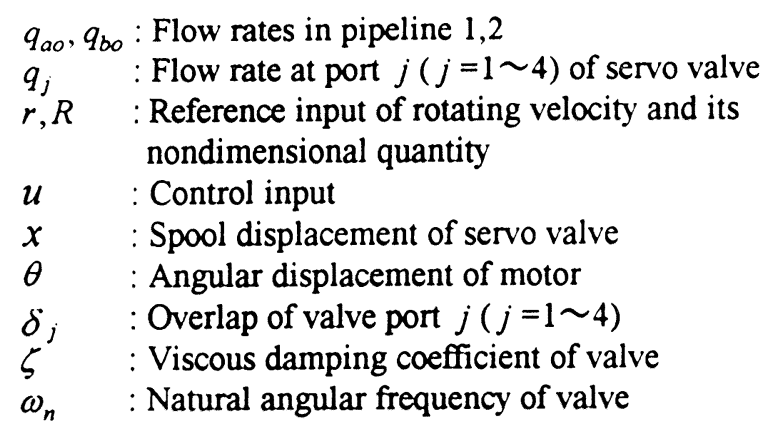

\section{INTRODUCTION}

In recent years, the fuzzy control algorithm is frequently applied to various kinds of systems for the reason of its simple algorithm, good adaptability to complex and nonlinear systems and so on. One of the problem when applying the fuzzy control algorithm is the tuning method of fuzzy control parameters for getting their optimal state.

This study deals with a self-tuning fuzzy controller (STFC) when it is applied to an electro-hydraulic servo system. The STFC in this study has a hierarchic structure consisting of an algorithm being identical to a fuzzy controller at the lower loop, and a tuning algorithm accommodating the performance evaluation and parameter modification function at the upper loop. As an algorithm for tuning at the upper loop, another fuzzy reasoning is adopted for constructing a fuzzy selftuner. The whole procedure of STFC is repeatedly performed until an acceptable level of control is achieved. The STFC must be able to access its performance in order to improve its control strategy. The basic functions of STFC can be summarized as follows: (a) to issue appropriate control action while evaluating the performance, (b) to modify the control action based on the evaluation.

It is verified by experiment and digital simulation that the STFC developed in this study is useful and effective for the control of electro-hydraulic servo system.

\section{SELF-TUNING FUZZY CONTROL SYSTEM}

\subsection{Constitution of System}

The schematic diagram of the electro-hydraulic servo system employed in this study is shown in Fig.l. Basically, the system consists of a servo valve, a hydraulic motor (gear motor: hereafter called motor for simplicity) and a microcomputer. The computer has three roles acting as a fuzzy controller, a fuzzy self-tuner and an acquisition system for detecting state variables of the control system. For the first role, the computer executes the fuzzy reasoning based on a feedback signal of rotating velocity of motor $n$, and then sends a control input $u$ to the servo amplifier.

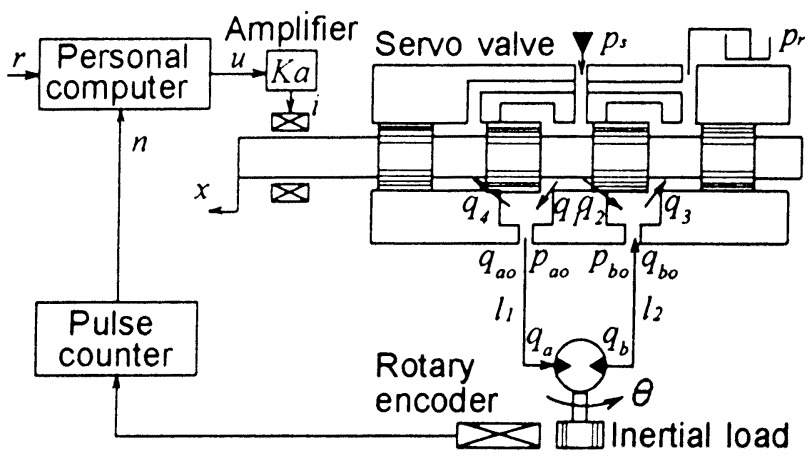

Fig. 1 Electro-hydraulic servo system

\subsection{Fuzzy Controller}

The controller for controlling the servo system in Fig. 1 is designed in the following manner by applying fuzzy reasoning.

Firstly, in order to execute the fuzzy reasoning with discretized fuzzy variables, a discretized set of integers $\{10,11, \cdots, 129,130\}$ is chosen as a support set of fuzzy variables as indicated in Fig.2. As for the linguistic elements, we chose five elements, as a lesser amount than conventional use, that is NB, NS, ZO, PS and PB. The adopted membership functions (MSF) are shown in Fig.2(a) and Fig.2(b) for the condition part and the operation part. respectively. In the condition part shown in Fig. 2(a), the grade of MSF is chosen as integers $\{0.1$. $\cdots, 99,100\}$. On the other hand, the grade of MSF for the operation part becomes unnecessary, since the MSF has a singleton as shown in Fig.2(b).

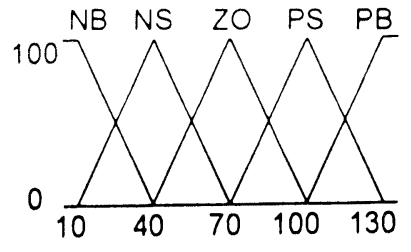

(a)Condition part

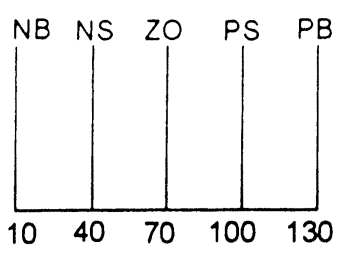

(b)Operation part
Fig. 2 Membership functions for controller

Secondly, the input and output variables for fuzzy reasoning are treated as follows. Two variables of rotating velocity error $e(=r-n)$ and change in error at one sampling time $\dot{e}$ are chosen as variables for the condition part. On the other hand, change in control input $\dot{u}$ is chosen as a variable for the operation part. The fuzzy rule thus constructed is shown in Table 1. 
Table 1 Fuzzy rules of controller

\begin{tabular}{|l|l|l|l|l|l|}
\hline$\dot{e}_{0}^{\text {eo }}$ & NB & NS & ZO & PS & PB \\
\hline PB & NB & NB & NB & PS & PB \\
\hline PS & NB & NB & NS & PS & PB \\
\hline$Z O$ & NB & NB & ZO & PB & PB \\
\hline NS & NB & NS & PS & PB & PB \\
\hline NB & NB & NS & PB & PB & PB \\
\hline
\end{tabular}

\section{NB=Negative Big} NS=Negative Small $\mathbf{Z O}=$ Zero PS=Positive Small PB=Positive Big
It is necessary for the variables $e$ and $\dot{e}$ to be normalized to match the support set $(10 \sim 130)$, when they are input to the fuzzy controller. The normalization of $e, \dot{e}$ into $e_{o}, \dot{e}_{o}$, respectively, is done by the next two equations.

$$
\begin{array}{ll}
e_{\circ}=A e+70 & ; 10 \leq e_{\circ} \leq 130 \\
\dot{e}_{o}=B \dot{e}+70 & ; 10 \leq \dot{e}_{o} \leq 130
\end{array}
$$

where, $A$ is error gain, $B$ is gain of change in error.

By inputting the normalized variables $e_{o}, \dot{e}_{o}$ to the fuzzy controller, the fuzzy reasoning can be executed based on the fuzzy rule given in Table 1. As the result, the change in control input $\dot{u}$ is generated. The control

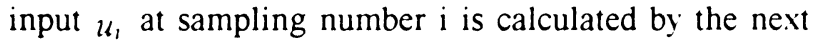
equation.

$$
u_{i}=u_{i-1}+C \dot{u}
$$

where, $C$ is control input gain.

In the operation of controller, three gains of $A, B, C$ have to be adjusted to their optimal values. As a method of parameter modification for $A, B, C$, we employ a selftuning algorithm based on fuzzy reasoning.

\subsection{Fuzzy Self-Tuner}

3.3.1 Constitution of self-tuner The role of the fuzzy self-tuner is to adjust three gains $A, B, C$ until an acceptable level of control is achieved. This adjustment is performed automatically by detecting the dynamic response of the system. For the evaluation of the system performance, we adopt three performance indexes, that is, a damping ratio $D$, an overshoot ratio $E$ and a rise time ratio $F$. These three indexes are defined as follows in terms of the step response curve shown in Fig. 3.

$$
D=\frac{e_{2}}{e_{3}}, \quad E=\frac{e_{1}}{r}, \quad F=\frac{t_{i}}{t_{i-1}}
$$

where, $t_{i}=t_{60} / t_{05}$ ( $\mathrm{i}$ : sampling number ), and the definition of $t_{60}$ and $t_{05}$ is indicated in Fig. 3.

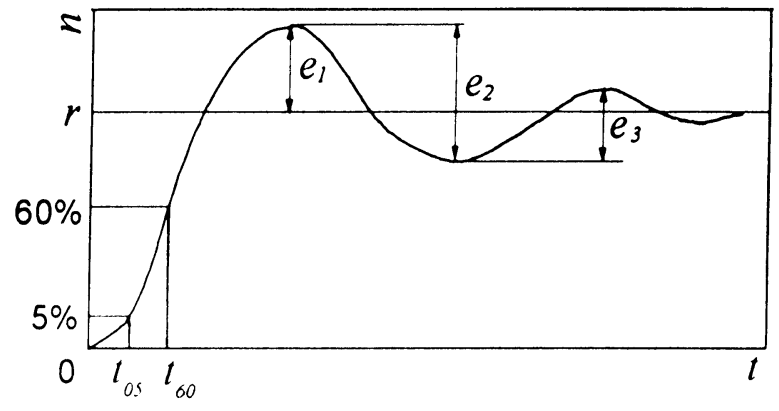

Fig.3 Performance indexes for step response

The estimation of the optimal values for gains $A, B, C$ is carried out by adopting the following evaluation function $I t a$, which is the integral of time multiplied absolute error.

$$
\text { It } a=\int t|n(t)-n(\infty)| d t
$$

By finding the minimum value of the function $I t a$, the parameter modification is evaluated to be accomplished.

The block diagram of the system implemented with the STFC is shown in Fig.4. As shown in the figure, the self-tuner executes three processes as follows

(1) The process for extracting three indexes $D, E, F$ from step response curves.

(2) The process for calculating the incremental factors $a, b, c$ for gains $A, B, C$, respectively, based on the indexes $D, E, F$. As a method for this calculation, an algorithm based on fuzzy reasoning is employed, which will be demonstrated later on.

(3) The process for calculating renewed gains for $A, B, C$ in terms of $a, b, c$

The optimal tuning will be accomplished by repeating the processes in the preceding

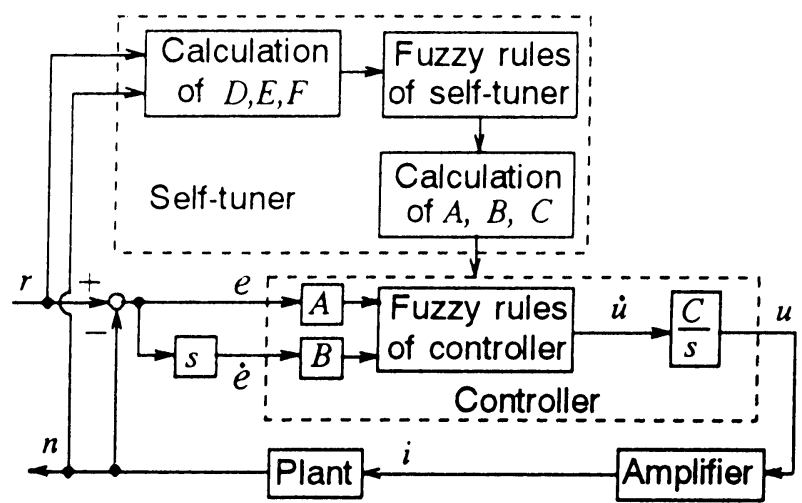

Fig.4 Fuzzy control system with a self-tuner 
3.3.2 Fuzzy reasoning for self-tuning As an algorithm for the self-tuner, fuzzy reasoning was adopted again as shown in Fig.4. The main reason for it is that the algorithm can be comparatively simple, by accommodating as many as common parts to the fuzzy controller. The followings are the main parts of this algorithm which was constituted independently from the fuzzy controller.

(1) The indexes $D, E, F$ are normalized by the next equations so as to match the support set $(10 \sim 130)$.

$$
\begin{array}{ll}
D_{O}=120 D+10 ; & 10 \leqq D_{0} \leqq 130 \\
E_{O}=400 E+10 ; & 10 \leqq E_{O} \leqq 130 \\
F_{O}=180(F-1)+70 ; & 10 \leqq F_{O} \leqq 130
\end{array}
$$

(2) The indexes Do,Eo,Fo bring about three variables for the condition parts. This fact forces us to employ three dimensional membership functions which will make the algorithm extremely complicated. In order to avoid this difficulty, we adopt a rather simplified method as follows, making use of features of fuzzy reasoning.

The method is a rather practical one to reduce three dimensional tuning rules to two dimensional ones. In practice. we adopted just two dimensional rules for the tuning of gains $A, B$, neglecting the rule influenced by the third dimension. On the other hand, two kinds of two dimensional rules were adopted for gain $C$. Thus obtained four rules are represented in Table 2. As shown in (a), (b) of Table 2, the gains $A, B$ are tuned by two condition part variables $D o, E o$. For the gain $C$, while. the rule based on $D o$ and $F_{O}$ is used as a dominant rule, by applying another rule based on Do,Eo as a compensation rule.

Table 2 Fuzzy rules of self-tuner

\begin{tabular}{|l|l|l|l|l|l|}
\hline EO & PB & PS & $Z O$ & NS & NB \\
\hline PB & NS & NS & $Z O$ & $Z O$ & PS \\
\hline PS & NS & NS & $Z O$ & $Z O$ & PS \\
\hline$Z O$ & $Z O$ & $Z O$ & $Z$ & ZO & PS \\
\hline NS & $Z O$ & $Z O$ & $Z O$ & $Z O$ & PS \\
\hline NB & $Z O$ & $Z O$ & $Z O$ & $Z O$ & PS \\
\hline
\end{tabular}

(a) Rules for $A$

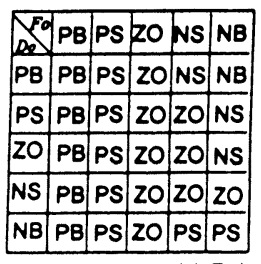

(c) Rules for
In this simplified algorithm, the choice of two variables for the condition parts, and further the determination of each rule were performed by the aid of experiment and simulation.

3.3.3 Modification of gains Modification of gains $A, B, C$ in each cycle $\mathrm{i}$ is performed in terms of the next equations.

$$
\begin{aligned}
& A_{1}=A_{i-1} \alpha^{a} \\
& B_{1}=B_{i-1} \beta^{b} \\
& C_{i}=C_{i-1} \gamma^{c}
\end{aligned}
$$

where, $\alpha, \beta, \gamma$ are the coefficients of modification.

\section{MATHEMATICAL MODEL FOR SIMULATION}

For a purpose to assist the design of self-tuner, together with for an evaluation of dynamic performance of the system, we adopt an analysis by digital simulation. A mathematical model for the simulation is derived under the following assumptions.

(1) The flow saturation existing in the characteristics of flow rate versus valve displacement in servo valve is taken into consideration.

(2) Overlaps in servo valve is taken into consideration.

(3) Each mathematical model of two pipelines between servo valve and gear motor is described by that of onedegree-of-freedom-system.

In the following, we derive relevant equations of the system in Fig.1.

The relation of servo amplifier is written as,

$$
i=k_{a} u
$$

The equation of motion for the valve spool is described as,

$$
\frac{d^{2} x}{d t^{2}}+2 \zeta \omega_{n} \frac{d x}{d t}+\omega_{n}{ }^{2} x=k_{\nu} \omega_{n}{ }^{2} i
$$

The inlet and outlet flow rates for the valve are calculated by the orifice equations:

$$
\begin{aligned}
& q_{1}=c_{s 1} w\left(x-\delta_{1}\right) \sqrt{\frac{2}{\rho}\left(p_{s}-p_{a o}\right)} \quad ; x>\delta_{1} \\
& q_{2}=c_{s 2} w\left(|x|-\delta_{2}\right) \sqrt{\frac{2}{\rho}\left(p_{s}-p_{b o}\right)} ; x<-\delta_{2}
\end{aligned}
$$




$$
\begin{array}{ll}
q_{3}=c_{s 3} w\left(x-\delta_{3}\right) \sqrt{\frac{2}{\rho} p_{b o}} & ; x>\delta_{3} \\
q_{4}=c_{s 4} w\left(|x|-\delta_{4}\right) \sqrt{\frac{2}{\rho} p_{a o}} & ; x<-\delta_{4}
\end{array}
$$

where, we put $q_{j}=0 \quad(\mathrm{j}=1 \sim 4)$ when the inequalities shown in the above equations are not satisfied.

Flow rates in the pipelines are written as,

$$
\begin{aligned}
& q_{a o}=q_{1}-q_{4} \\
& q_{b o}=q_{3}-q_{2} \\
& q_{a o}-q_{a}=\frac{\pi d_{1}^{2} l_{1}}{4 K} \frac{d p_{a o}}{d t} \\
& q_{b}-q_{b o}=\frac{\pi d_{2}^{2} l_{2}}{4 K} \frac{d p_{b o}}{d t}
\end{aligned}
$$

Flow rates in the gear motor are given as,

$$
q_{a}=q_{b}=\frac{v_{m}}{2 \pi} \frac{d \theta}{d t}
$$

The rotating velocity of gear motor is expressed as,

$$
n=\frac{60}{2 \pi} \frac{d \theta}{d t}
$$

The equation of motion for the gear motor is written as,

$$
I_{m k} \frac{d^{2} \theta}{d t^{2}}+b_{m} \frac{d \theta}{d t}+f_{m}=\frac{v_{m}}{2 \pi}\left(p_{a o}-p_{b o}\right) ; k=1 \sim 3
$$

The digital simulation of the system is carried out by using Eq.(12) to Eq.(24).

\section{RESULTS OF EXPERIMENT \& SIMULATION}

The dynamic performances of the STFC-system is investigated by experiment and digital simulation. The procedures of experiments for attaining the optimal tuning of the gains $A, B, C$ consists of three processes as follows.

First, we set the initial values of the gains $A, B, C$ as arbitrary values. Next, the reference step input of

\begin{tabular}{|c|c|}
\hline $\begin{array}{l}\text { Servo } \\
\text { valve }\end{array}$ & $\begin{array}{l}\mathcal{w}=34.6 \mathrm{~mm}, k_{v}=1.158 \mathrm{~mm} / \mathrm{A} \\
\omega_{n}=1.28 \times 10^{3} \mathrm{rad} / \mathrm{s}, \zeta=0.7 \\
\delta_{1}=6.8 \times 10^{-5} \mathrm{~m}, \delta_{2}=5.8 \times 10^{-5} \mathrm{~m} \\
\delta_{3}=6.8 \times 10^{-5} \mathrm{~m}, \delta_{4}=4.4 \times 10^{-5} \mathrm{~m}\end{array}$ \\
\hline $\begin{array}{l}\text { Gear } \\
\text { motor }\end{array}$ & $\begin{array}{ll}\nu_{m}=6.3 \mathrm{~cm}^{3} / \mathrm{rev} \\
\text { Large load: } \quad \operatorname{Iml}=5.73 \times 10^{-3} \mathrm{~kg} \cdot \mathrm{m}^{2} \\
\text { Medium load: } & \operatorname{Im}_{m}=3.15 \times 10^{-3} \mathrm{~kg} \cdot \mathrm{m}^{2} \\
\text { Small load: } & I_{m 3}=4.25 \times 10^{-4} \mathrm{~kg} \cdot \mathrm{m}^{2}\end{array}$ \\
\hline $\begin{array}{l}\text { Other } \\
\text { parame- } \\
\text { ters }\end{array}$ & $\begin{array}{l}l_{1}=l_{2}=41 \mathrm{~cm}, d_{1}=d_{2}=9.4 \mathrm{~mm} \\
k_{a}=63.8 \mathrm{~mA} / \mathrm{V}, p_{s}=2.94 \mathrm{MPa} \\
\rho=855 \mathrm{~kg} / \mathrm{m}^{3}, K=1.6 \mathrm{GPa}\end{array}$ \\
\hline
\end{tabular}
magnitude $R$ is input to the system. Subsequently the resultant response data of rotating velocity of the motor $N$ is sent to the computer and the self-tuner. Finally the self-tuner modifies the gains $A, B, C$. The whole these processes are repeated automatically until the optimal tuning is attained. The main parameters of the system used in the experiments are summarized in Table 3.

Table 3 Main parameters of system

As indicated in Table 3, we used three kinds of inertial loads, that is, large, medium and small ones. In the first place, the experimental results obtained with the medium load are demonstrated in the followings. Figure 5 shows a series of step responses for a reference input of $R=2$ $(r=1000 \mathrm{rpm})$. In this series of experiments from No.1run to No.9, the optimal tuning was evaluated to be performed on No.9-run. In the figure, nevertheless, only four responses on No.1, 3, 6 and 9 are indicated for a sake of simplicity. Together with the experimental results, simulated results are shown in the figure for a comparison. In the upward part of each figure, the values of gains $A, B, C$ and the value of the evaluation function I $t a$ on each run are indicated. From the results shown in Fig.5, we can state that the quality of responses are improved gradually with the change in gains $A, B, C$. From the response on No.9-run, we can observe that the performance of the system is considerably satisfactory. This fact verifies that the self-tuning has been well attained. From the comparison between experiment and simulation, furthermore, we can say that the two results agree with each other considerably well. As a result we can confirm the usefulness of the self-tuner developed in this study.

The results shown in Fig. 5 were obtained under the condition that the initial values of gains $A, B, C$ were set as comparatively small values and thus the responses in the initial stage appeared as moderate curves. In contrast to this condition, the initial values of gains $A, B, C$ were set as comparatively large values in the next place. Thus obtained result are shown in Fig.6. It is observed from the figure that the responses in the initial stage behave oscillatory motion. Nevertheless we can confirm that the self-tuner acts effectively even in such a severe condition.

Under another conditions, for examples, when the load and the supply pressure are changed independently, 


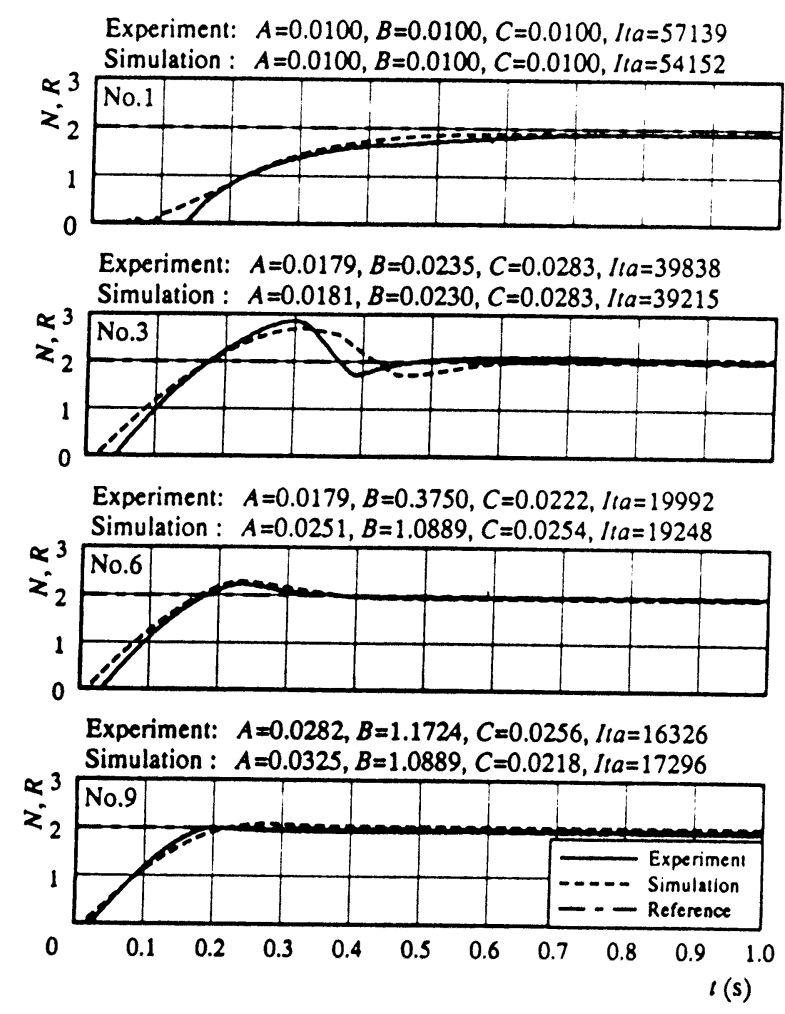

Fig.5 Step responses (medium load)

\section{CONCLUSIONS}

A self-tuning fuzzy controller (STFC) was designed by applying it to an electro-hydraulic servo system. The parameters for self-tuning are, in this study, three gains, that is, each gain of error, change in error and control input. As a method of parameter modification, we employed a self-tuning algorithm based on fuzzy reasoning. This modification is repeatedly performed by

Experiment: $A=0.1000, B=0.5000, C=0.0500, I t a=62056$ Simulation : $A=0.1000, B=0.5000, C=0.0500$, tha $=27354$

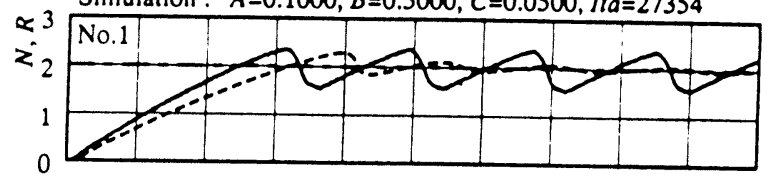

Experiment: $A=0.0891, B=1.2599, C=0.0445, l t a=27186$ Simulation : $A=0.1000, B=0.9615, C=0.0500, I_{t} a=25479$

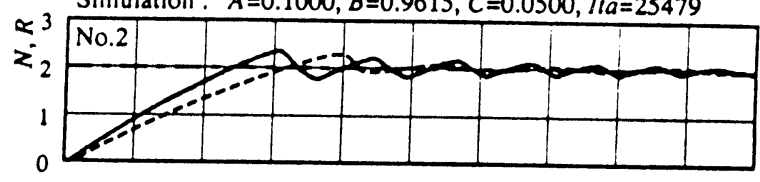

Experiment: $A=0.0788, B=3.0212, C=0.0437, I t a=19731$ Simulation: $A=0.1000, B=1.7092, C=0.0500, / t a=20751$

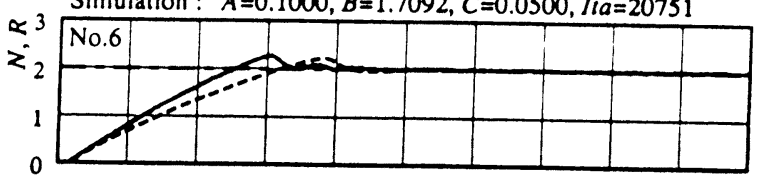

Experiment: $A=0.0812, B=5.7358, C=0.0618, / t a=17739$ Simulation : $A=0.1035, B=2.4172, C=0.0500, I t a=20122$

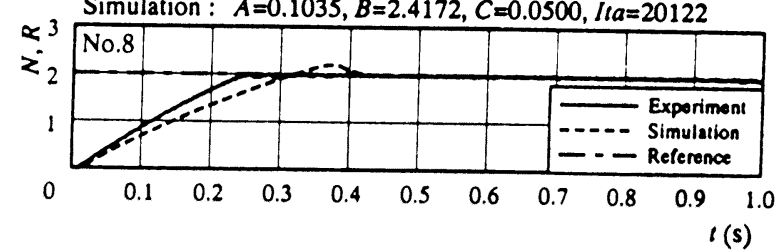

Fig.6 Step responses (large load)

detecting the dynamic response of the system until an acceptable level of control is achieved.

From the results of experiments and digital simulations, it was verified that the quality of responses was improved gradually with the change in three gains. As a consequence, it was confirmed that the STFC developed in this study is useful and effective for the control of electro-hydraulic servo system.

\section{REFERENCES}

1. Maeda and Murakami, Self-Tuning Fuzzy Controller, Trans. of the Society of Instrument and Control Engineers, 1988,24-2,pp191-197.

2. Q.Wang,A.Luo and Y.Lu, Intelligent Control for Electrohydraulic Proportional Cylinder, Fluid Power Edited by T.Maeda, 1993,pp309-313.

3. Nomura,Saito,Yokokawa,Takahashi and Sugano, An Auto-Tuning System Based on Fuzzy Reasoning for PID Controllers, Hitachi Review, 1989,71-8,ppl15-122.

4. Iwazaki,Morita and Maruyama, Plant Identification with Fuzzy Inference and Its Application to Auto-Tuning, Transactions of the Japan Society of Mechanical Engineers (C), 1992,58-554,pp2997-3002.

5. Hiraku,Muto and Yamada, Fuzzy Control of Electrohydraulic Servo System, Transactions of the Japan Society of Mechanical Engineers (C), 1992,58-555,pp3285-3290. 